\title{
FROM ASTRONOMY TO THE CLINIC: ADAPTIVE OPTICS BASED VISUAL SIMULATORS
}

. Maria Viñas - https://doi.org/10.1051/epn/2020302

\begin{abstract}
Adaptive optics based visual simulators with deformable mirrors, spatial light modulators or optotunable lenses are increasingly used to simulate vision through different complex multifocal lens designs. Their final utility in the clinic relies on their capability to predict visual performance after an ocular surgery.
\end{abstract}

\section{From Astronomy to vision}

Adaptive Optics (AO), a technology originally developed to image stellar objects with ground-based telescopes eliminating the degrading effects of the atmospheric turbulence, has more recently expanded applications into ophthalmology [1].

The eye is an optical instrument that projects scenes of the visual world onto the retina. However the human eye is far from being a perfect optical system, and, as a consequence, the images projected on the retina are blurred by diffraction, scattering and ocular aberrations. Since $\mathrm{AO}$ was first applied to the eye[2], its use to measure, correct or induce ocular aberrations has increased dramatically, and it has even made its way into the clinic. Wavefront sensing and $\mathrm{AO}$ have been applied to the understanding of the optical quality of the eye, and its change with refractive error, accommodation, aging in the normal eye, diseases and treatment (refractive surgery, cataract surgery, contact lenses, etc).

Conversely, AO has allowed probing the visual system under manipulated optics, either with fully corrected optical aberrations [3], through the optical aberrations of another subject or scaled/rotated versions of their own [4], or a phase pattern simulating a given correction (i.e. an intraocular lens (IOL), contact lens (CL) or a corneal treatment)[5]. AO visual simulators are particularly attractive to test vision in patients with new optical designs, prior to delivering surgical corrections to the patient or even manufacturing the lenses.

Providing patients the visual experience before implanting an IOL or fitting a CL is particularly relevant for multifocal corrections for presbyopia (the age-related loss of the ability to dynamically focus near and far objects). Multifocal corrections work under the principle of simultaneous vision, projecting simultaneously focused and defocused images on the retina. These corrections generally provide multifocality at the expense of reducing optical quality at all distances. Visual simulators allow undertaking systematic studies of visual performance testing multiple lens designs (programmable in the adaptive optics active element), which can be directly compared by the patient. As clinical instruments, $\mathrm{AO}$ visual simulators can help demonstrating the patient the experience of multifocality and can guide the patient and eye care practitioner in the selection of the most suitable correction.

\section{Adaptive Optics based visual simulators} In $\mathrm{AO}$ based visual simulators, an active optical element, typically a deformable mirror (DM) or a spatial light modulator (SLM), reproduces the equivalent phase map of a certain optical design in a plane conjugate to the subject's pupil plane, while the observer is looking at a visual stimulus. DMs allow simulating smooth optical designs, or to induce certain amounts of aberrations, while controlling the aberrations of the subject. In contrast, spatial light modulators (SLMs) are capable of reproducing abrupt phase maps due to their high spatial resolution, and to increase the effective phase range through the use of wrapped phase representations. Moreover, static simulation of a multifocal design can be obtained by placing the real IOL in a cuvette filled with water in a conjugate pupil plane projected in the eye.

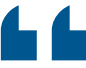

As clinical instruments, $\mathrm{AO}$ visual simulators can help demonstrating the patient the experience of multifocality and can guide the patient and eye care practitioner in the selection of the most suitable correction. 77 
A novel approximation to simultaneous vision simulation is the use of optotunable lenses working in temporal multiplexing mode (SimVis technology) [6]. These custom electronically driven lenses can produce fast periodic foci variations at speeds greater than the flicker fusion threshold of the human visual system, delivering seemingly static images on the subject's retina that emulate the effect of the multifocal correction. The simulation of multifocal corrections relies on evaluating the through focus energy distribution of the correction, from the knowledge of the spatially varying pupillary power distribution, and programming in the optotunablelens the corresponding time-varying focus changes.

AO visual simulators based on different active optical elements are increasingly used to simulate vision through different multifocal lens designs. An example of these type of systems is the multi-channel polychromatic AO visual simulator of the Visual Optics and Biophotonics lab (Madrid, Spain). The visual stimulus in this system is seen (by a camera or a patient) through different active optical elements in 4 separate channels: (1) a reflective deformable mirror (DM); (2) a reflective phase-only spatial light modulator (LCoS-SLM), (3) a simultaneous vision simulator based on temporal multiplexing of an opto-tunable lens (SimVis); and (4) a real IOL in a cuvette. The DM, SLM, SimVis and real IOL in a cuvette are placed in conjugate pupil planes of the system and allow visual simulation of complex multifocal designs.

An interesting question is whether this simulation correspond with the vision obtained through the physically manufactured real IOL tested on the same eye [7]. Comparison, on bench and in patients, in the same multi-channel AO visual simulator, of through focus (TF) optical and visual quality produced by real multifocal IOLs (M-IOLs) and their corresponding visual simulations using different active optical elements can help answering it. Optical quality metrics obtained from on-bench TFE-letter images (1P) and in vivo TF decimal Visual Acuity (VA) on patients shows similar TF visual performance (Figure 1) for a bifocal refractive M-IOL. TF curves show a general good correspondence between the through-focus performance with the real and simulated M-IOLs, both optically (on bench) and visually (measured VA in patients).Visual simulations in an $\mathrm{AO}$ system capture largely the optical and visual performance obtained with projected real and simulated IOLs.

\section{From the lab to the clinic: predicting post-surgery vision}

Reflective-DM or SLM-based visual simulators are mostly limited to experimental environments, given their relatively high complexity and dimensions, although some have made their way into commercial products. In these devices the visual experience is limited to stimuli projected in a display, subtending a relatively small visual field, in many cases monocularly. Ideal visual simulators in a clinical environment should be see-through, allowing a direct view of the real world and should display a larger visual field. Moreover, the ultimate utility of visual simulators, no matter the technology behind, relies on their capability to allow patients to experience vision before IOL implantation, since tested IOLs are designed to replace the natural crystalline lens. For that reason, to evaluate the limits of a clinical visual simulator it is necessary to compare directly the in vivo predicted and post- operative through focus visual quality in patients.

Figure 2 shows the pipeline of the validation process of a wearable see-through binocular clinical visual simulator (SimVis Gekko, 2Eyes Vision, Madrid, Spain) developed for

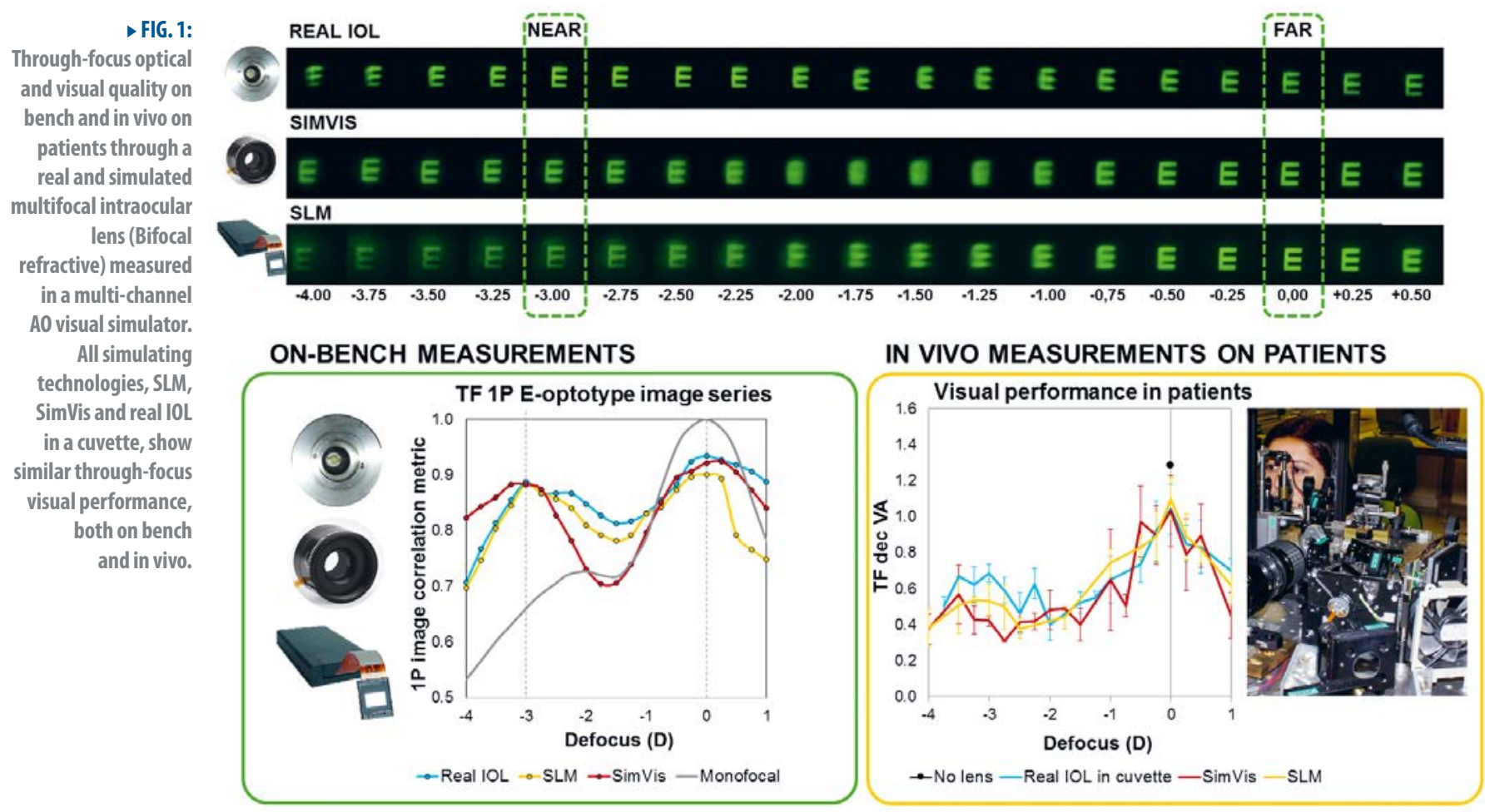



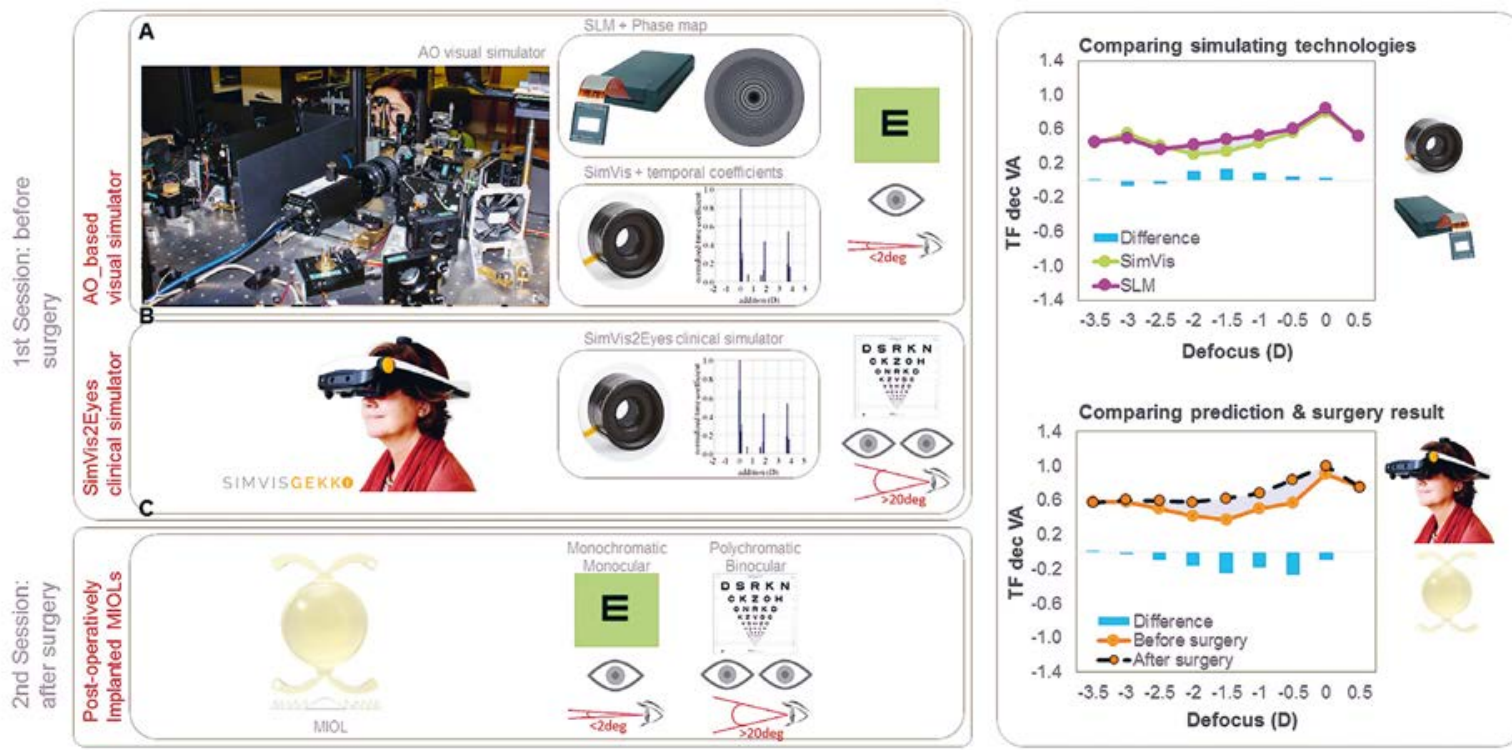

4 FIG. 2: From A0 based multi-channel visual simulator to a clinical simulator useful to predict post-surgery visual performance. Presurgery predictions match closely aftersurgery through focus visual performance.

the pre-surgical simulation of presbyopic corrections[8], where in vivo pre-operative simulated TF visual performance is compared with post-operative vision after implantation of a M-IOL in patients[9]. The comparison is made in patients that are implanted with diffractive trifocal lenses (the FineVision PODF, by PhysIOL), using two different simulating technologies (SLM and SimVis technology) and 2 different simulation platforms, an AO based visual simulator and the clinical simulator. When comparing the different simulations techniques pre-surgery, a similar TF visual performance is found between the TF VA with the SLM (pink line) and the SimVis (green line) simulation in the AO platform. The clinical simulator based on SimVis technology (orange line) predicts well the post-surgery TF performance (black dotted line).

In general, visual simulators are able to predict the relative multifocal performance of a specific IOL design, since TF visual performance pre-surgery (simulated IOL) and TF visual performance after-surgery (real IOL) show good correspondence. Visual simulations are useful programmable tools to predict the relative visual performance with multifocal IOLs, both in an AO environment and in a large field of view clinical binocular device.

\section{Acknowledgements}

M.Viñas thanks her co-authors Clara Benedí-García, Sara Aissati, Daniel Pascual, Vyas Akondi, Carlos Dorronsoro and Susana Marcos from Spanish National Research Council, and Mercedes Romero, Francisco Poyales, and Nuria Garzón from IOA Madrid Innova Ocular.

\section{Funding \& Financial disclosure}

European Research Council (ERC-2011-AdC 294099); H2020 Marie Skłodowska-Curie Actions (H2020 COFUND Marie Curie 291820 program); Spanish Goverment (FIS2014-56643R, FIS2017-84753R, FPU16/01944, ISCIII DTS16/00127); Private company PhysIOL (Collaborative agreement).
SimVis technology is protected by 4 licensed patents: P200930055, P201331436, P201531397 \& P201730854

MV and some of her co-authors (Daniel Pascual, Vyas Akondi, Carlos Dorronsoro and Susana Marcos) have financial interest in the company 2EyesVision, proprietary of SimVis technology

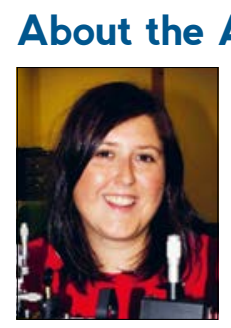

Author

Maria Viñas is a senior postdoctoral researcher at the Visual Optics and Biophotonics Group of the Institute of Optics of the Spanish National Research Council (CSIC). She is founding partner of 2EyesVision, a spin-off company proprietary of SimVis technology.

\section{References}

[1] S. Marcos et al., Vision research 132, 3 (2017)

[2] Liang, D. R. Williams, and D. T. Miller, Journal of the Optical Society of America. A, Optics, image science and vision 14, 2884 (1997)

[3] S. Marcos, L. Sawides, E. Gambra, and C. Dorronsoro, Journal of vision 8, 11 (2008)

[4] P. Artal, L. Chen, E. J. Fernandez, B. Singer, S. Manzanera, and D. R. Williams, Journal of vision 4, 281 (2004)

[5] M. Viñas, C. Dorronsoro, A. Radhakrishnan, C. Benedí-García, E. A. LaVilla, J. Schwiegerling, and S. Marcos, Biomedical optics express 8, 2055 (2017)

[6] C. Dorronsoro, X. Barcala, E. Gambra, V. Akondi, L. Sawides, Y. Marrakchi, V. Rodriguez-Lopez, C. Benedí-García, M. Viñas, E. Lage, and S. Marcos, Optics express 27, 2085 (2019)

[7] M. Viñas, C. Benedí-García, S. Aissati, D. Pascual, V. Akondi, C. Dorronsoro, and S. Marcos, Scientific reports 9, 1539 (2019)

[8] C. Dorronsoro, J. R. Alonso-Sanz, D. Pascual, M. Velasco-Ocaña, P. Perez-Merino, and S. Marcos, Optica 3, 918 (2016)

[9] M. Viñas, S. Aissati, M. Romero, C. Benedí-García, N. Garzon, F. Poyales, C. Dorronsoro, and S. Marcos, Biomedical optics express 10, 5801 (2019) 